\title{
Generation of Soliton Molecules with Independently Evolving Phase in a Mode-Locked Fiber Laser
}

Article in Optics Letters · May 2010

DOI: 10.1364/OL.35.001578 · Source: PubMed

CITATIONS

37

8 authors, including:

\section{Bülend Ortaç}

Bilkent University

121 PUBLICATIONS 1,690 CITATIONS

SEE PROFILE

Jens Limpert

Friedrich Schiller University Jena 728 PUBLICATIONS $\quad 10,947$ CITATIONS

SEE PROFILE
READS

157

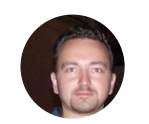

Oleg Egorov

Friedrich Schiller University Jena

96 PUBLICATIONS 731 CITATIONS

SEE PROFILE

\section{Andreas Tünnermann}

Fraunhofer Institute for Applied Optics and Pre... 944 PUBLICATIONS 11,451 CITATIONS

SEE PROFILE

Some of the authors of this publication are also working on these related projects:

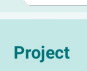
Spatially resolved 3D measurement of long-period gratings written by fs-laser inscription in large mode area fibers View project

All content following this page was uploaded by Oleg Egorov on 03 October 2017. 


\title{
Observation of soliton molecules with independently evolving phase in a mode-locked fiber laser
}

\author{
Bülend Ortaç, ${ }^{1,5, *}$ Alexandr Zaviyalov, ${ }^{2,6}$ Carsten K. Nielsen, ${ }^{1,3}$ Oleg Egorov, ${ }^{2}$ Rumen Iliew, ${ }^{2}$ \\ Jens Limpert, ${ }^{1}$ Falk Lederer, ${ }^{2}$ and Andreas Tünnermann ${ }^{1,4}$ \\ ${ }^{1}$ Institute of Applied Physics, Friedrich-Schiller-Universität Jena, Max-Wien-Platz 1, 07743 Jena, Germany \\ ${ }^{2}$ Institute of Condensed Matter Theory and Solid State Optics, Friedrich-Schiller-Universität Jena, Max-Wien-Platz 1, \\ 07743 Jena, Germany \\ ${ }^{3}$ University of Aarhus, Department of Physics, DK-8000 Aarhus, Denmark \\ ${ }^{4}$ Fraunhofer Institute for Applied Optics and Precision Engineering, 07745 Jena, Germany \\ ${ }^{5}$ Present address, UNAM-Institute of Materials Science and Nanotechnology, Bilkent University, \\ 06800 Ankara, Turkey \\ ${ }^{6}$ aleksandr.zavyalov@uni-jena.de \\ *Corresponding author: ortac@unam.bilkent.edu.tr
}

Received February 12, 2010; revised April 1, 2010; accepted April 6, 2010; posted April 9, 2010 (Doc. ID 124141); published May 5, 2010

\begin{abstract}
We report the experimental generation of two-soliton molecules in an all-polarization-maintaining ytterbium-doped fiber laser operating in the normal dispersion regime. These molecules exhibit an independently evolving phase and are characterized by a regular spectral modulation pattern with a modulation depth of $80 \%$ measured as an averaged value. Moreover, the numerical modeling confirms that the limited modulation depth of the spectrum is caused by the evolution of the phase difference between the pulses. (C) 2010 Optical Society of America

OCIS codes: $140.3510,190.4370,190.5530$.
\end{abstract}

The generation of soliton molecules, also frequently termed bound states, in mode-locked fiber lasers attracted a great deal of interest because of their potential applications in communication lines, optical logic systems, and high-resolution optics. Usually such molecules consist of two pulses and are characterized by the peak-to-peak separation $\rho$ and the phase difference $\phi$ between both pulses. Theoretically, a few kinds of stable scalar soliton molecules have been discovered in mode-locked fiber lasers, namely, molecules with an invariant phase [1,2], or molecules which are slightly vibrating or shaking around a stationary state $[2,3]$, and molecules with an independently evolving phase [4-7] and such with a flipping phase [6]. Experimentally, only soliton molecules with an invariant phase [5,8-10], with a rotating phase [5], and vibrating molecules [11] have been reported to date. This may be attributed to practical difficulties in the measurement of minor changes in molecule parameters or of the complex internal dynamics like periodic or chaotic evolution of the phase difference between the pulses.

In the present Letter we report what is to the best of our knowledge the first experimental observation of robust two-soliton molecules with an independently evolving phase in a mode-locked fiber laser. The laser operates in the normal dispersion regime [12] and is based on a polarization-maintaining (PM) fiber that guarantees the scalar nature of the solitons. A distinct feature of these molecules is the regularly modulated spectrum with a modulation depth significantly less than $100 \%$. The experimental results are confirmed by numerical modeling backing the genuine observation of this type of soliton molecule.

0146-9592/10/101578-3/\$15.00
The experimental configuration of the passively mode-locked wave-breaking-free fiber laser is shown in Fig. 1 (for details see [13]). All-fiber components are based on the PM single-clad concept. A section of $31 \mathrm{~cm}$ highly $\mathrm{Yb}$-doped fiber is spliced between the identical lengths of passive fiber-based components. Light from the pump diode is coupled into the fiber using a thin-film wavelength division multiplexing coupler. A fiber pig-tailed thin-film 30/70 coupler is inserted in this configuration to monitor the laser operation and to select the polarization axis of the PM fiber resonator. This scalar platform is a very attractive configuration to study soliton molecule dynamics since a single polarization state propagates inside the cavity. Passive mode-locking is achieved by using a high-modulation depth (30\%) and short relaxation time ( $\sim 500 \mathrm{fs})$ saturable absorber mirror (SAM). The passive fiber length inside the cavity is $5.6 \mathrm{~m}$. A 1250 lines/mm highly efficient transmission grating-pairbased dispersion delay line (DDL) ensures partial compensation of the dispersion of the fiber. A halfwave plate is used between the gratings and the PM fiber to ensure the excitation of a field polarized along the slow axis. In our experiment, the distance between the gratings is $1.6 \mathrm{~cm}$, which leads to a total round-trip positive group delay dispersion of $+0.03 \mathrm{ps}^{2}$ at $1035 \mathrm{~nm}$. Thus the laser operates in the

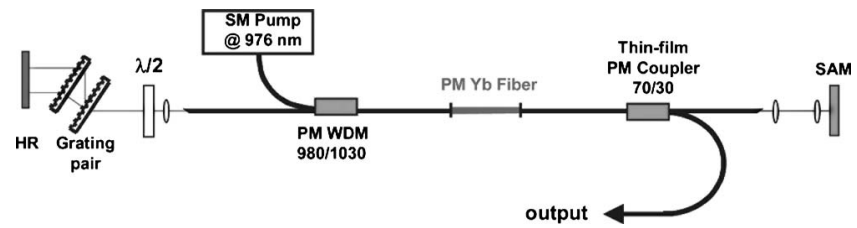

Fig. 1. Experimental setup of the mode-locked fiber laser. 
normal dispersion regime. The output signal is analyzed by a commercial autocorrelator and an optical spectrum analyzer (Ando AQ6315A) requiring a few minutes to record a spectrum. A $50 \mathrm{GHz}$ oscilloscope (Tektronix TDS 8000) and a $25 \mathrm{GHz}$ high-speed photodetector (New Focus 1434) are used to study the time evolution of the laser output.

The typical operation of this laser in the single pulse regime is obtained for about $94 \mathrm{~mW}$ pump power, and positively chirped output pulses with an autocorrelation width of $8.2 \mathrm{ps}$ (full width at halfmaximum) are generated [13]. With our high-speed oscilloscope and autocorrelator we have verified that there is no multiple pulsing per round trip. When the pump power reaches $125 \mathrm{~mW}$, the laser delivers twopulse molecules. Figure 2(a) shows the autocorrelation trace of such a soliton molecule. The separation distance between the pulses is $18.7 \mathrm{ps}$, which equals the delay time from the central- to the side-peaks in the figure. Figure 2(b) shows the optical spectrum of the soliton molecule, which is strongly modulated as a direct consequence of the coherence of the pulses. The modulation period is $0.19 \mathrm{~nm}$, which corresponds to a peak separation of $18.7 \mathrm{ps}$. It is important to note that the spectrum is regularly modulated with just
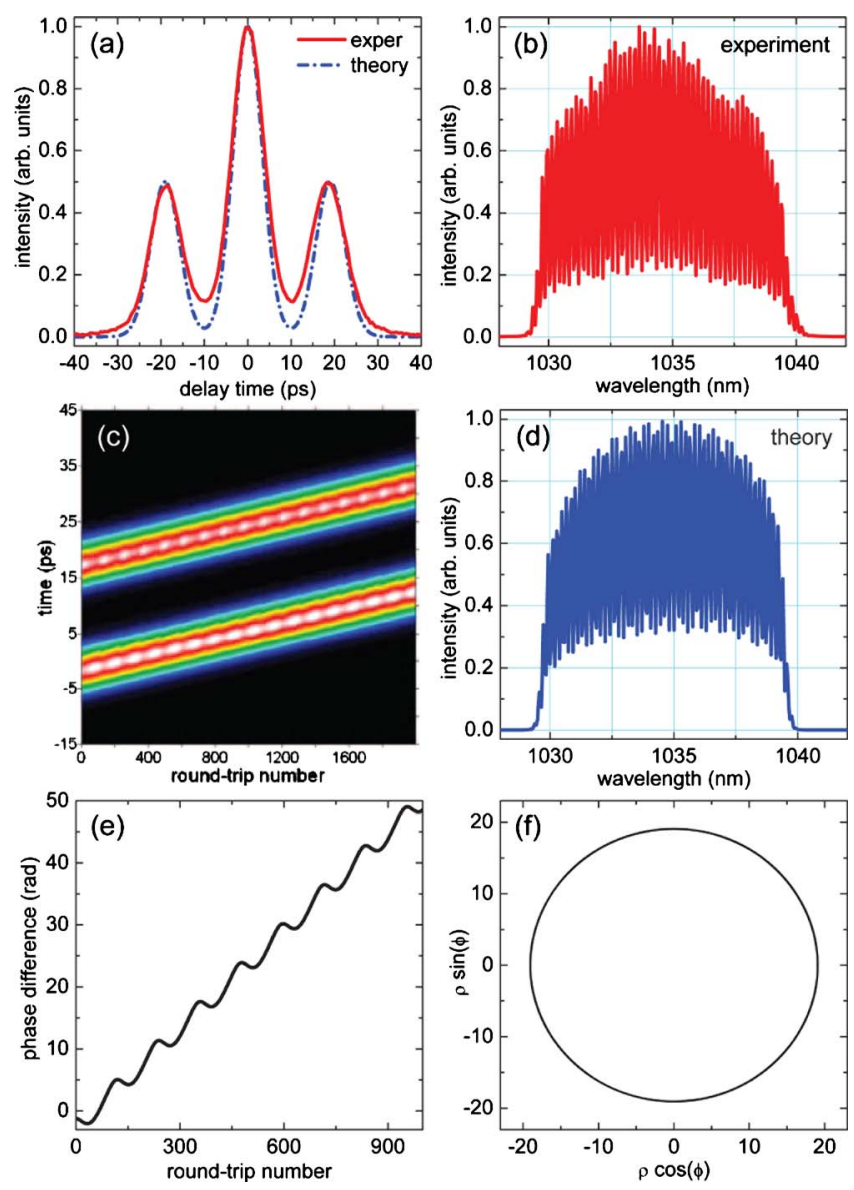

Fig. 2. (Color online) (a) Experimentally measured and numerically calculated intensity autocorrelation functions and optical spectrum [(b) experiment; (d) modeling] of a soliton molecule. Other modeling results: (c) evolution of the molecule, (e) the phase difference between pulses as a function of the round-trip number, and (f) evolution trajectory in the phase plane.
$80 \%$ of the modulation depth caused by the averaging effect over many pulses in the experimental measurement. Moreover, especially this unmodulated part of the spectrum allows us to conclude that we observe the soliton molecule with an evolving phase. These results differ from previous works where fully modulated spectra were reported that correspond to twopulse molecules with a fixed separation distance and an invariant phase relation between pulses $[10,14]$. Simultaneously, our results show significant differences from those for the vibrating and rotating phase molecules, which exhibit a blurred modulated [11] or an unmodulated spectrum [5], respectively.

In order to understand the influence of the averaging effect on the measured spectral profile we consider the simple example of two co-propagating Gaussian pulses. When the pulses propagate with both invariant $\rho$ and $\phi$, then we obtain the typical modulated profile with $100 \%$ of the modulation depth independent of the averaging of the spectral intensity. For an oscillating phase difference as $\phi(z)=\phi_{0}$ $+A_{\Phi} \sin (z)$ and a fixed separation the averaging of the spectral intensity over one period leads to a decrease in the modulation depth in dependence on $A_{\Phi}$ [see Figs. 3(a)-3(c)]. Obviously, at each instant the modulation depth of the spectrum is $100 \%$. But after averaging it can be significantly reduced due to the shift of the spectral modulation caused by the phase evolution $\phi(z)$. Namely, we can obtain an almost unmodulated spectrum for certain values $A_{\Phi}$, which reproduces results in [5].

For a constant phase difference but an oscillating separation as $\rho(z)=\rho_{0}+A_{P} \sin (z)$ the averaged spectrum is irregularly modulated depending on $A_{P}$ [see Figs. 3(d)-3(f)]. The heterogeneity appears due to the averaging of a 100\% modulated spectrum but with different modulation periods, since the separation $\rho(z)$ defines the modulation frequency. Hence, the averaged spectral profile contains important informa-
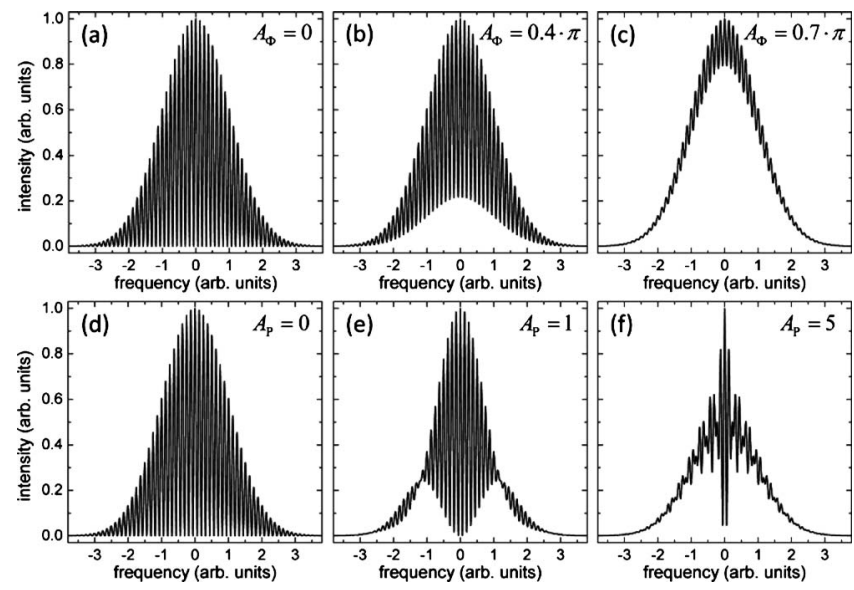

Fig. 3. Influence of the averaging process on the measured spectral profile in dependence on the internal molecule dynamics for two Gaussian pulses of duration $T_{0}$ and separation distance $\rho_{0}=25 T_{0}$. In (a)-(c) the phase difference between pulses is oscillating with an amplitude $A_{\Phi}$ and the separation is fixed. In (d)-(f) the separation is oscillating with an amplitude $A_{P}$ (normalized with $T_{0}$ ) and the phase difference is fixed. 
tion on the internal dynamics of soliton molecules. Consequently, from our experimentally obtained spectrum with $80 \%$ modulation depth we can conclude that the phase difference between the pulses is evolving with the number of round trips. These considerations can also describe the spectrum reported for the vibrating molecule [11], which is a result of both phase and separation oscillations.

We confirm our claim that the laser works in the independently evolving phase regime by numerical simulations. We use a lumped model where all individual elements are separately described. The fiber components and the DDL are described by a modified nonlinear Schrödinger equation [2],

$$
\begin{aligned}
& \frac{\partial U(z, t)}{\partial z}+\frac{i}{2}\left(\beta_{2}+i g(z) T_{1}^{2}\right) \frac{\partial^{2} U(z, t)}{\partial^{2} t} \\
& =\frac{g(z) U(z, t)}{2}+i \gamma|U(z, t)|^{2} U(z, t),
\end{aligned}
$$

where $U(z, t)$ is the envelope of the pulse, $z$ is the propagation coordinate, $t$ is the retarded time, $\beta_{2}$ is the second-order dispersion, $\gamma$ represents the fiber nonlinearity, and $T_{1}$ is the dipole relaxation time. $g(z)$ is the gain of the doped fiber which is assumed to be saturable [2]. For the SAM we use the AgrawalOlsson model [15],

$$
\begin{gathered}
\frac{\partial U(z, t)}{\partial z}=-\frac{1}{2} \delta(z, t) U(z, t), \\
\frac{\partial \delta(z, t)}{\partial t}=\frac{\delta_{0}-\delta(z, t)}{T_{\text {relax }}}-\frac{\delta(z, t)|U(z, t)|^{2}}{E_{\text {sat }}^{\mathrm{SAM}}},
\end{gathered}
$$

where $\delta(z, t)$ is the loss introduced by the absorber, $\delta_{0}$ is the small-signal loss, $T_{\text {relax }}$ is the recovery time, and $E_{\mathrm{sat}}^{\mathrm{SAM}}$ is the saturation energy. According to the experiment we set $\beta_{2}=0.024 \mathrm{ps}^{2} \mathrm{~m}^{-1}$ and $\gamma$ $=0.0052 \mathrm{~W}^{-1} \mathrm{~m}^{-1}$ for the passive fiber and assume for the doped fiber $E_{\mathrm{sat}}^{\mathrm{Gain}}=0.5 \mathrm{~nJ}$ (gain saturation), $g_{0}$ $=2.76 \mathrm{~m}^{-1}$ (small-signal gain), and $T_{1}=69 \mathrm{fs}$. The length of the absorber and the small-signal loss were adjusted to a modulation depth of $30 \%$, the SAM relaxation time is $600 \mathrm{fs}$, and $E_{\mathrm{sat}}^{\mathrm{SAM}}=6 \mathrm{pJ}$. The output loss is equal to $30 \%$. The total cavity dispersion is reduced by the DDL and amounts to $0.05 \mathrm{ps}^{2}$.

The numerical results are shown in Fig. 2. The spectrum was calculated as an average over many periods of the phase oscillations. Excellent agreement between calculated and measured optical spectra can be recognized. Specifically, experiments and simulations yield spectra of equal width, the same parabolic shape, and similarly regular modulation. There is a minor difference in the modulation depth (experiment $\approx 80 \%$ and simulations $\approx 70 \%$ ). The intensity profile of the simulated molecule is almost identical to that being experimentally observed. This is proven by almost identical autocorrelation functions. Using the inherent advantages of modeling properties can be identified as the temporal evolution of this molecule, the evolution trajectory on the phase plane, and the phase difference between pulses as a function of the round-trip number. The numerically obtained circular evolution trajectory on the phase plane is a consequence of the infinitely growing phase difference at an almost constant peak separation. The phase $\phi$ is growing because of slightly different amplitudes of the leading and trailing pulses according to the mechanism explained in [6]. Unfortunately this difference of amplitudes cannot be recognized in both the experimental and the simulated autocorrelation functions for its smallness, but it can be clearly seen from the simulated propagation of the soliton molecule in Fig. 2(c). Thus, the numerical results reproduce the experimental ones with a high accuracy and confirm that the obtained soliton molecule exhibits an independently evolving phase which causes the partly unmodulated averaged spectrum profile.

In conclusion we experimentally demonstrated and theoretically explained the generation of scalar soliton molecules in a normal dispersion mode-locked fiber laser. These molecules have an independently evolving phase, which results in an incomplete modulation of the optical spectrum. The simple example of two Gaussian pulses confirms that the limited spectral modulation depth is a direct consequence of the particular phase evolution. Moreover, numerical simulations nearly perfectly reproduce the experimental results and prove that these molecules have an independently evolving phase.

This work was supported by the Deutsche Forschungsgemeinschaft (DFG) (research unit 532).

\section{References}

1. N. N. Akhmediev, A. Ankiewicz, and J. M. Soto-Crespo, Phys. Rev. Lett. 79, 4047 (1997).

2. A. Zavyalov, R. Iliew, O. Egorov, and F. Lederer, Phys. Rev. A 79, 053841 (2009).

3. J. M. Soto-Crespo, Ph. Grelu, N. N. Akhmediev, and N. Devine, Phys. Rev. E 75, 016613 (2007).

4. J. M. Soto-Crespo and N. N. Akhmediev, J. Opt. Soc. Am. B 16, 674 (1999).

5. M. J. Lederer, B. Luther-Davies, H. H. Tan, C. Jagadish, N. N. Akhmediev, and J. M. Soto-Crespo, J. Opt. Soc. Am. B 16, 895 (1999).

6. A. Zavyalov, R. Iliew, O. Egorov, and F. Lederer, Phys. Rev. A 80, 043829 (2009).

7. A. Zavyalov, R. Iliew, O. Egorov, and F. Lederer, Opt. Lett. 34, 3827 (2009).

8. D. Y. Tang, B. Zhao, D. Y. Shen, C. Lu, W. S. Man, and H. Y. Tam, Phys. Rev. A 66, 033806 (2002).

9. Ph. Grelu, F. Belhache, F. Gutty, and J. M. Soto-Crespo, Opt. Lett. 27, 966 (2002).

10. A. Hideur, B. Ortaç, T. Chartier, M. Brunel, H. Leblond, and F. Sanchez, Opt. Commun. 225, 71 (2003).

11. M. Grapinet and P. Grelu, Opt. Lett. 31, 2115 (2006).

12. T. Schreiber, B. Ortaç, J. Limpert, and A. Tünnermann, Opt. Express 15, 8252 (2007).

13. C. K. Nielsen, B. Ortaç, T. Schreiber, J. Limpert, R. Hohmuth, W. Richter, and A. Tünnermann, Opt. Express 13, 9346 (2005).

14. B. Ortaç, A. Hideur, M. Brunel, C. Chédot, J. Limpert, A. Tünnermann, and F. Ö. Ilday, Opt. Express 14, 6075 (2006).

15. G. Agrawal and N. Olsson, IEEE J. Quantum Electron. QE-25, 2297 (1997). 